\title{
Artigo Original / Original Paper Nuevo registro de Melocactus peruvianus (Cactaceae) y estado de conservación del género Melocactus en el Ecuador
}

New record of Melocactus peruvianus (Cactaceae) and conservation status of the Melocactus genus in Ecuador

\author{
Christian R. Loaiza Salazar ${ }^{1,4}$ \& Natalia Molina-Moreira ${ }^{2,3}$
}

\begin{abstract}
Resumen
Los cactus globulares son el grupo de cactáceas en mayor riesgo de extinción en el Ecuador Continental. Melocactus bellavistensis, se encuentra distribuido en un valle seco interandino en la provincia de Loja y actualmente se encuentra listado en la categoría de Datos Insuficientes (DD) por la UICN. La otra especie, M. peruvianus se conoce solo en dos localidades confirmadas en Huaquillas (El Oro), cerca de la frontera con Perú y está considerada como extinta en el Ecuador. Se reporta un nuevo registro de M. peruvianus en un pequeño remanente de Espinar Litoral dentro de la Reserva Ecológica Arenillas. Se analiza la distribución y probabilidad de ocurrencia para ambas especies, y se estima el área de distribución que ocupan mediante el método del polígono mínimo convexo (PMC). Se recomienda una nueva evaluación del estado de conservación y se proponen dos nuevos tipos de categorías para ambas especies en Ecuador. Finalmente, se presentan varias alternativas para su conservación.
\end{abstract}

Palabras clave: bosque seco, categorías, distribución, evaluación, pligro crítico.

\begin{abstract}
The globular cacti are the group of cacti that are most at risk of extinction in Continental Ecuador. Melocactus bellavistensis, is distributed in a dry inter-Andean valley in the province of Loja and is currently listed in the category of Deficient Data (DD) by the IUCN. The other species, M. peruvianus is known only in two confirmed localities in Huaquillas (El Oro), near the border with Peru and is considered extinct in Ecuador. A new record of M. peruvianus is reported in a small remnant of Espinar Litoral within the Arenillas Ecological Reserve. The distribution and occurrence probability for both species is analyzed, and the home range was estimated by the use of the polygon minimum convex method (PMC). We recommended a new assessment of the conservation status and two new types of categories are proposed for both species in Ecuador. Finally, several alternatives are presented for its conservation.
\end{abstract}

Key words: dry forest, categories, distribution, evaluation, critical endangered.

\section{Introducción}

El género Melocactus Link \& Otto (1827) actualmente está representado por 50 especies y subespecies de cactus globulares originarios de México, las Indias Occidentales y de la parte norte de Sudamérica (Britton \& Rose 1922; Madsen 1989; Anderson 2001; Hunt et al. 2006; Machado 2009). Este género se encuentra presente en varios países tanto de América Central como en América del Sur, incluyendo algunas islas del Caribe como Cuba, en donde se pueden encontrar once especies (Taylor 1991); sin embargo, su centro de mayor endemismo y diversidad se encuentra al este de Brasil, especialmente en el estado de Bahía (Lambert et al. 2006). Taylor \& Zappi (2004) reconocen 22 especies y subespecies, de las cuales 18 especies y seis subespecies se consideran endémicas. Machado (2009) incrementa el número

\footnotetext{
${ }^{1}$ Universidad Nacional Mayor de San Marcos, Instituto de Ciencias Biológicas "Antonio Raimondi", Facultad de Ciencias Biológicas, Germán Amézaga No. 375, Lima, Perú.

${ }^{2}$ Universidad Espíritu Santo, km 2.5 vía a Samborondón, Guayaquil, Ecuador.

${ }^{3}$ ORCID: 0000-0002-8197-1137

${ }^{4}$ Autor para correspondencia: cactus_ecuador@hotmail.com
} 
de especies en Brasil, señalando un total de 27 especies, de las cuales 26 especies y subespecies son endémicas para el país.

Este género también se encuentra presente en algunos países como El Salvador y otros países de la región (Britton \& Rose 1922). En Colombia el género está representado por cinco especies y siete subespecies (Fernández-Alonso \& Xhonneux 2002). Los registros más australes del género se encuentran en Ecuador y Perú (Madsen 1989; Ostolaza 2014). En Ecuador se reconocen solamente dos especies: Melocactus bellavistensis Rauh \& Backeberg, 1957 presente en los valles secos interandinos de la provincia de Loja (Loaiza 2008, 2010) y Melocactus peruvianus Vaupel, 1913 reportado en algunas localidades dentro de la provincia de El Oro (Madsen 2002; Cerón et al. 2006).

La conservación de este género no ha sido evaluada correctamente en el Ecuador debido a la poca información disponible sobre su distribución y el estado actual de sus poblaciones. En el caso de $M$. bellavistensis, la IUCN ubica a esta especie dentro de la categoría de Datos Insuficientes (DD), debido a la falta de información sobre su estado poblacional a nivel de Perú (Ostolaza \& Loaiza 2017a). Su estado poblacional en Ecuador es bastante reducido, señalándose una disminución del $30 \%$ de sus poblaciones en los últimos 10 años, por lo cual se considera que esta especie se encuentra en estado Vulnerable (VU). En el caso de $M$. peruvianus, la evaluación realizada por la IUCN ubica a esta especie dentro de la categoría de Preocupación Menor (LC), debido a su amplia distribución, su abundancia local y su presencia en varias áreas protegidas (Ostolaza \& Loaiza 2017b); sin embargo, se considera que esta especie se encuentra extinta en Ecuador debido a los pocos registros existentes dentro de su área de distribución (Jørgensen \& León-Yánez 1999; Madsen \& Montúfar 2011).

Este estudio analiza la distribución del género Melocactus en el Ecuador, se reporta un nuevo registro de M. peruvianus en la provincia de El Oro y se proponen algunas medidas de conservación para ambas especies.

\section{Materiales y Métodos}

\section{Área de estudio}

La fase de campo se realizó en la Reserva Ecológica Arenillas (Fig. 1). Esta área protegida por el estado ecuatoriano, se encuentra localizada entre los cantones Arenillas y Huaquillas, dentro de la provincia de El Oro, al suroeste del Ecuador, entre las coordenadas $03^{\circ} 31^{\prime} 06.4^{\prime \prime}$ ' y $80^{\circ} 07^{\prime} 54.1^{\prime \prime} \mathrm{W}$. La temperatura dentro de la Reserva varía entre 24 y $30^{\circ} \mathrm{C}$. La época de lluvia se presenta entre los meses de Enero y Abril, con un rango de precipitación entre $500-1.000 \mathrm{~mm} /$ año y la época seca ocurre entre los meses de Mayo-Diciembre. El rango altitudinal registrado va de 6 a $110 \mathrm{msnm}$. La información relacionada con la distribución de $M$. bellavistensis fue recopilada por el autor principal de este trabajo durante el periodo 2007-2010 (Loaiza 2008, 2010).

\section{Análisis distributivo}

Para obtener la distribución espacial de ambas especies se utilizó el algoritmo de Máxima Entropía Maxent, el cual permite ubicar las zonas con mayor probabilidad de presencia de una especie y delimitar su área de distribución geográfica (Phillips et al. 2006; Phillips \& Dudík 2008). Para el desarrollo del modelo se utilizó un total de 80 registros en el caso de $M$. bellavistensis, y de 23

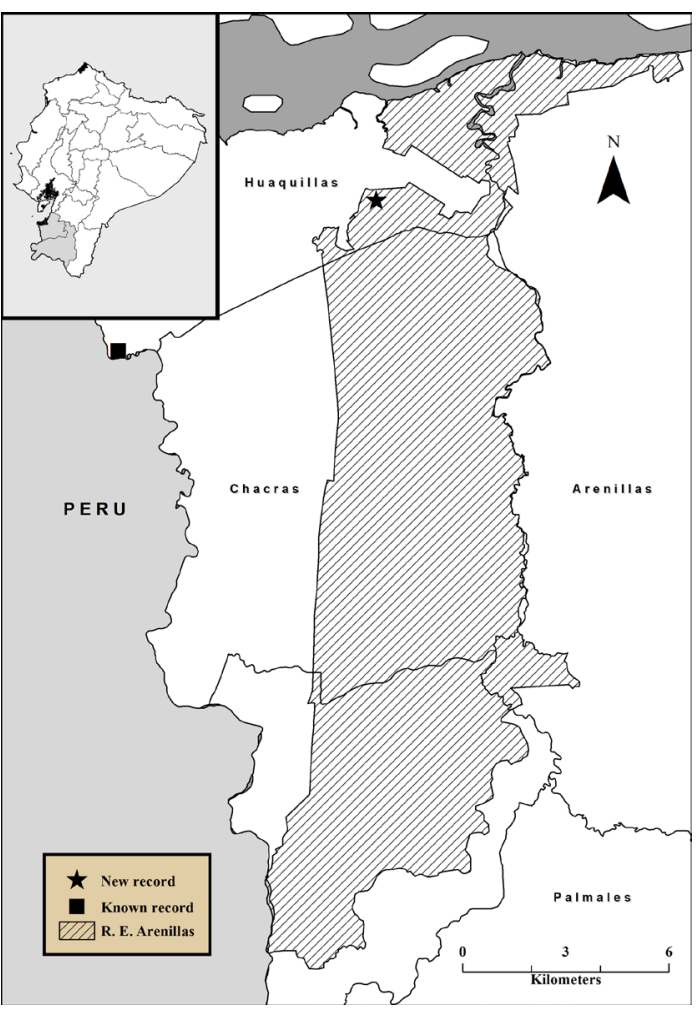

Figura 1 - Área de estudio y nuevo registro de Melocactus peruvianus dentro de la Reserva Ecológica Arenillas / Ministerio del Ambiente del Ecuador (2017). Figure 1 - Study area and new record of Melocactus peruvianus within the Arenillas Ecological Reserve / Ministerio del Ambiente del Ecuador (2017). 
registros en el caso de $M$. peruvianus (Ecuador/ Perú). Ambos modelamientos fueron obtenidos desarrollando 10 repeticiones, con el $25 \%$ de los registros usados como test data, 500 iteraciones y 10.000 puntos de respaldo con la finalidad de obtener una mayor precisión en el modelamiento. Los modelamientos fueron obtenidos con base a la probabilidad de presencia de cada especie,

Para estimar el área de distribución se utilizó una herramienta disponible en ArcGis llamada Xtools Pro, la cual permite crear polígonos con base a los registros de una especie y estimar su área de vida en $\mathrm{km}^{2}$. Los mapas finales fueron realizados con el programa ArcGis 10.3.

\section{Resultados}

\section{Melocactus bellavistensis}

\section{Distribución y hábitat}

Esta especie se encuentra ampliamente distribuida a nivel de la provincia de Loja. Ha sido registrada en varias zonas de bosque seco interandino dentro de los cantones: Catamayo (El Boquerón, El Tambo y Trapichillo), Calvas (Hda. Bella María), Gonzanamá (Nambacola), y en Paltas (San Vicente del Río). Su área de vida comprende las laderas de montaña y zonas rocosas situadas a lo largo de la cuenca del río Catamayo y sus principales afluentes (Madsen 1989; Loaiza 2010). Según el sistema de clasificación vegetal propuesto por Valencia et al. (1999), esta especie ocupa las siguientes formaciones vegetales: Bosque semideciduo montano bajo de los andes occidentales, Bosque siempreverde montano bajo de los andes occidentales y Bosque deciduo piemontano de la costa. Su rango altitudinal se sitúa entre 500-1.500 msnm (Taylor 1991; Jørgensen \& León-Yánez 1999; Loaiza et al. 2009).

En su hábitat natural suele crecer ya sea en forma solitaria o formando pequeñas agrupaciones de entre 3 y 4 individuos (Fig. 2) (Loaiza 2008), entre zonas rocosas o bajo sombra, generalmente junto a árboles de faique (Acacia macracantha), casi siempre en asociación con algunas especies como Agave americana, Croton wagneri, Mimosa quitensis, Opuntia quitensis, y solamente en muy raras ocasiones con Armatocereus laetus, siendo los lugares con sombra en donde mejor se desarrolla (Loaiza 2010). De acuerdo a los registros obtenidos en varias localidades de estudio durante el período 2007-2010, se considera que antiguamente esta especie debió ser muy común dentro de toda su área de distribución, siendo actualmente muy difícil de registrar, debido al intenso proceso de extracción ilegal del cual ha sido objeto por parte del ser humano.

Algunas de las poblaciones más importantes de las que se tiene registro están ubicadas en zonas restringidas y de difícil acceso dentro de las localidades antes mencionadas. Es posible que existan remanentes de poblaciones en zonas limítrofes con la República del Perú, especialmente en el sector de Yaramine (Sozoranga), pero siempre dentro del mismo tipo de formación vegetal. Muchas de las zonas en las cuales su presencia debió ser bastante común actualmente están muy alteradas, debido a actividades antropógenas como la expansión de áreas para la agricultura y ganadería, pérdida de la cobertura vegetal original, introducción de especies exóticas, minería, entre otros factores que han contribuido a reducir en gran número su estado poblacional.

\section{Modelamiento predictivo}

El modelamiento realizado para esta especie tuvo un nivel de aceptación bastante óptimo

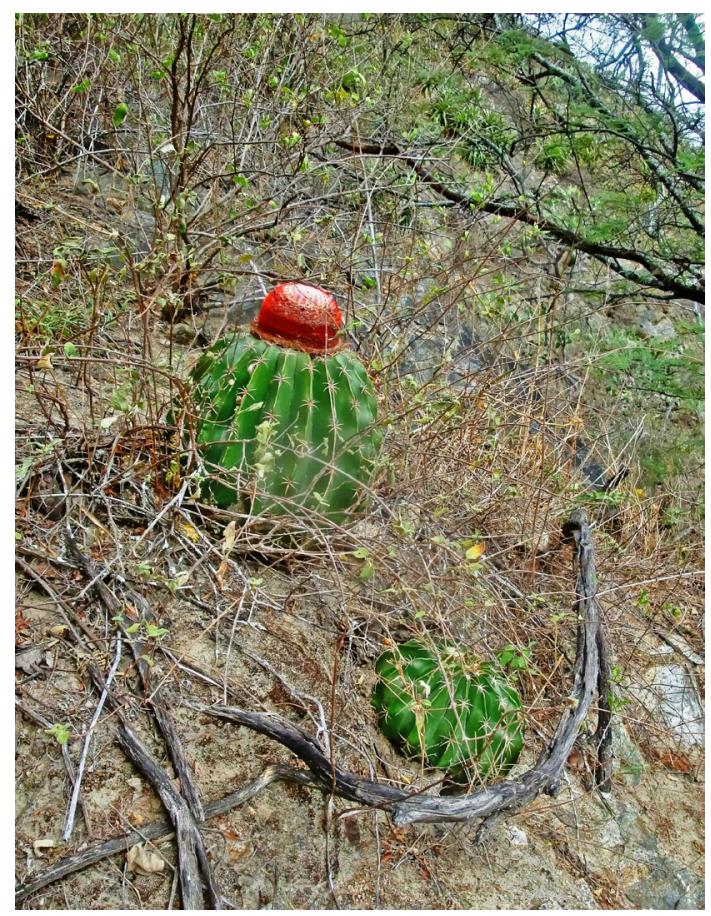

Figura 2 - Melocactus bellavistensis en su hábitat natural. Foto: Christian R. Loaiza Salazar.

Figure 2-Melocactus bellavistensis in its natural habitat. Photo: Christian R. Loaiza Salazar. 
(Fig. 3). El valor de sensibilidad obtenido en el test data fue de 0.982 , lo cual indica que el $98,2 \%$ de las localidades observadas / datos de presencia estarían incluidos en la predicción. El valor de AUC obtenido en el modelamiento final fue de $0.986(98,6 \%)$, lo cual indica un nivel de confianza igualmente aceptable. De las variables utilizadas, diez variables con valores superiores al $1 \%$ contribuyeron con el modelamiento, lo cual representa el 98,5\% de la varianza explicada (Tab. 1). Las variables ambientales con mayor influencia para la distribución de $M$. bellavistensis fueron: Temperatura media del cuatrimestre más frío $(36,7 \%)$, Precipitación del periodo más lluvioso $(18,7 \%)$ y Precipitación del período más seco $(17,4 \%)$. Otras variables de mayor y menor importancia se señalan en la Tabla 1.

\section{Estado de conservación}

La distribución de esta especie ha sido bastante analizada y estudiada en algunos trabajos anteriores (Loaiza 2008, 2010). Según la información disponible, se considera que su

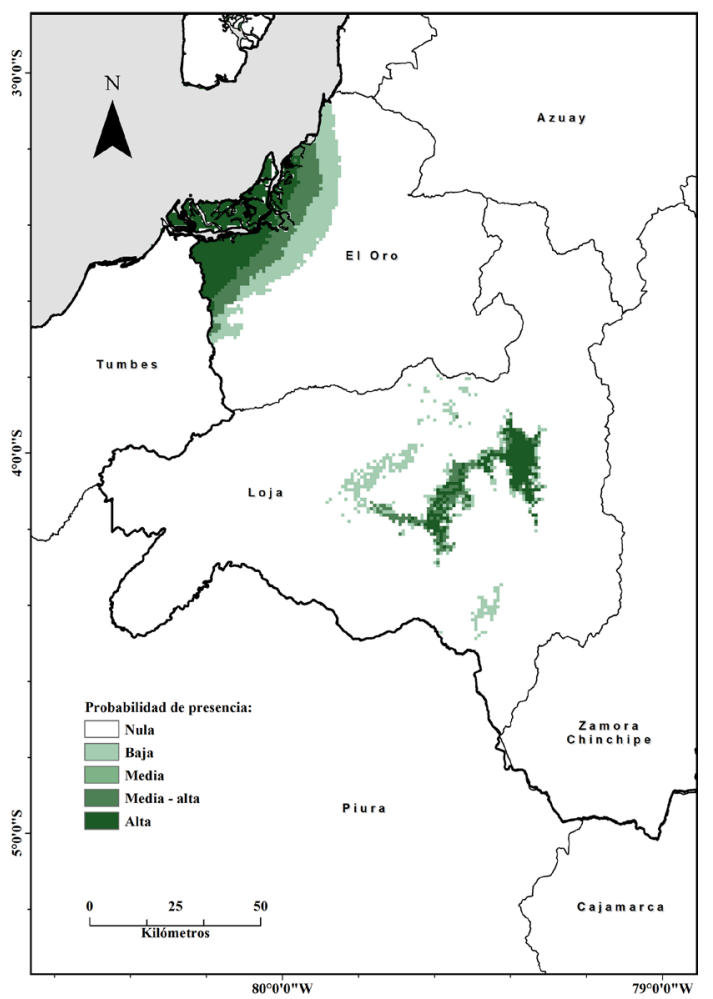

Figura 3 - Modelamiento predictivo del género Melocactus.

Figure 3 - Predictive modeling of the Melocactus genus. área de distribución es de $711,3 \mathrm{~km}^{2}$ (Fig. 4) sin embargo, de acuerdo a un censo realizado durante el período 2007-2010, se considera que su estado poblacional no superaría los 500 individuos (Loaiza obs. per). De acuerdo a la poca literatura existente que aporta datos sobre el estado de conservación de esta especie, se considera que las poblaciones más afectadas son las que se encuentran ubicadas a nivel del cantón Catamayo, debido a lo fácil que resultaba extraer las plantas de su hábitat natural para su posterior venta en los distintos mercados locales (Madsen 1989; Loaiza 2008).

Según algunas encuestas realizadas entre 1988 y 1989 por Jens E. Madsen (Herbario Loja), se sabe que en aquel tiempo las plantas obtenidas de los bosques eran comercializadas en los principales mercados locales del cantón Catamayo, a un precio no mayor a lo que actualmente equivaldrían $\$ 5$. Afortunadamente, las poblaciones que se encuentran ubicadas dentro del cantón Calvas (Hda. Bella María) y en Paltas (El Guato), están situadas en zonas

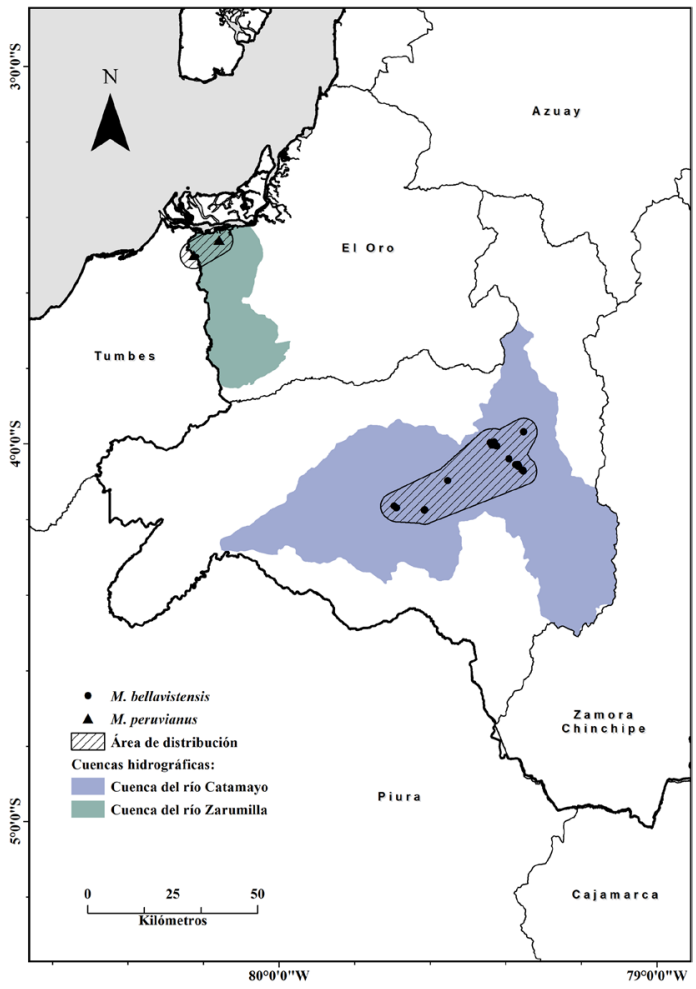

Figura 4 - Área de distribución (PMC) del género Melocactus.

Figure 4 - Home range (PMC) of the Melocactus genus. 
Tabla 1 - Porcentaje de contribución (\% C) e importancia de la permutación (PI) de cada variable para el modelamiento predictivo logístico y potencial de Melocactus bellavistensis y M. peruvianus.

Table 1 - Percent contribution (\% C) and permutation importance (PI) of each variable for the potential and logistic modeling of Melocactus bellavistensis and M. peruvianus.

\begin{tabular}{|c|c|c|c|c|c|}
\hline \multirow[t]{3}{*}{ Variables } & \multirow{3}{*}{ Descripción } & \multicolumn{4}{|c|}{ Modelamiento } \\
\hline & & \multicolumn{2}{|c|}{ M. bellavistensis } & \multicolumn{2}{|c|}{ M. peruvianus } \\
\hline & & $\% \mathrm{C}$ & PI & $\% \mathrm{C}$ & PI \\
\hline bio_1 & Temperatura media anual & 2.7 & 0 & 0 & 0 \\
\hline bio_2 & Oscilación diurna de la temperatura & 1.7 & 4.5 & 3.2 & 0 \\
\hline bio_3 & Isotermalidad & 0 & 0 & 0.4 & 0 \\
\hline bio_4 & Estacionalidad de la temperatura (\%) & 2.6 & 25.7 & 3.0 & 2.4 \\
\hline bio_5 & Temperatura máxima del período más cálido & 0.4 & 49.3 & 0.9 & 0.1 \\
\hline bio_6 & Temperatura mínima del período más frío & 2.3 & 0 & 2.9 & 0 \\
\hline bio_7 & Oscilación anual de la temperatura & 5.5 & 4.4 & 2.4 & 3.9 \\
\hline bio_8 & Temperatura media del cuatrimestre más lluvioso & 0 & 0 & 0.6 & 1.8 \\
\hline bio_9 & Temperatura media del cuatrimestre más seco & 0.3 & 0.1 & 0 & 0.6 \\
\hline bio_10 & Temperatura media del cuatrimestre más cálido & 0.5 & 0 & 0.3 & 0 \\
\hline bio_11 & Temperatura media del cuatrimestre más frío & 36.7 & 0 & 0 & 0 \\
\hline bio_12 & Precipitación anual (mm) & 0.1 & 0 & 20 & 6 \\
\hline bio_13 & Precipitación del período más lluvioso & 18.7 & 3.2 & 0.4 & 33.8 \\
\hline bio_14 & Precipitación del período más seco & 17.4 & 0.2 & 0.5 & 0 \\
\hline bio_15 & Estacionalidad de la precipitación (\%) & 0 & 0 & 0.4 & 0 \\
\hline bio_16 & Precipitación del cuatrimestre más lluvioso & 0 & 0 & 2.9 & 0.2 \\
\hline bio_17 & Precipitación del cuatrimestre más seco & 0.4 & 0 & 56.8 & 48.6 \\
\hline bio_18 & Precipitación del cuatrimestre más cálido & 9.7 & 12.6 & 4.4 & 0.8 \\
\hline bio_19 & Precipitación del cuatrimestre más frío & 1.2 & 0.1 & 1.1 & 1.9 \\
\hline Total & & 100 & - & 100 & - \\
\hline
\end{tabular}

restringidas, de difícil acceso y bastante alejadas de las áreas urbanas (Fig. 3), lo cual evitó que sean igualmente explotadas por el ser humano. Además, se considera que la actividad comercial de estas plantas ha disminuido, probablemente por lo difícil que resulta poder obtenerlas actualmente, debido a la intensa depredación de la cual fueron objeto en años anteriores.

Otro factor que también ha ocasionado graves impactos para la supervivencia de esta especie ha sido, sin duda, la falsa creencia que se tiene acerca de sus "supuestas propiedades alucinógenas", lo cual ha motivado a que ciertas personas, principalmente shamanes, utilicen esta especie en sus rituales curativos (Kvist \& Moraes 2006). Hasta la presente fecha, no se conoce de ningún estudio científico que haya comprobado realmente tales propiedades. Esta creencia probablemente se originó debido a que el nombre vernáculo de $M$. bellavistensis en la 
provincia de Loja es "Cactus San Pedro", lo cual seguramente hizo que algunas personas asociaran erróneamente a esta especie con el famoso cactus "San Pedrillo" (Echinopsis pachanoi), el cual si posee tales propiedades.

Esta especie se encuentra actualmente asignada por la IUCN dentro de la categoría de Datos Insuficientes (DD) (Ostolaza \& Loaiza 2017a). Nosotros proponemos que en Ecuador esta especie debe ser incluida dentro de la categoría de especies En Peligro (EN), debido a una reducción del $50 \%$ de su tamaño poblacional en los últimos 10 años (A2acd) y por su limitado rango geográfico en base al criterio B2ab i-v.

\section{Melocactus peruvianus}

Distribución y hábitat

La distribución de esta especie ocurre en zonas costeras a nivel de la provincia de El Oro (Arenillas y Huaquillas), dentro de las formaciones vegetales correspondientes a Matorral seco de tierras bajas de la costa y Zona de Manglar (Valencia et al. 1999) (Fig. 3). El primer registro conocido de esta especie en el Ecuador fue realizado por Edward F. Anderson (Taylor 1991). El último registro del que se tiene conocimiento fue realizado en la Reserva Ecológica Arenillas, dentro de una pequeña zona de Espinar Litoral en la Reserva Ecológica Arenillas (Cerón et al. 2006).

\section{Nuevo registro}

Durante un estudio de evaluación de la estructura de la flora leñosa para la zonación de ecosistemas de la Reserva Ecológica Arenillas, se realizó un muestreo en un área de $20.500 \mathrm{~m}^{2}$ y se diferenciaron los siguientes ecosistemas: Bosques de Manglar y Salinas, Bosque Seco de Conchales, Espinar Litoral, Bosque Seco de Planicies, Bosque Seco de Colinas y Herbazales (Molina-Moreira 2017). En el ecosistema de Espinar Litoral, en un rango altitudinal de 10-20 msnm, se registró una población de $M$. peruvianus, con una densidad poblacional de seis individuos en $1.000 \mathrm{~m}^{2}$, ubicado junto a la camaronera Cayancas (Fig. 5). Esta nueva población se encuentra ubicada en las siguientes coordenadas: $03^{\circ} 27^{\prime} 36.5^{\prime \prime} \mathrm{S}$ y $80^{\circ} 09^{\prime} 30.4^{\prime \prime} \mathrm{W}$, a una distancia de $142,8 \mathrm{~m}$ del registro realizado por Cerón et al. (2006). Con base a este nuevo registro, la superficie que ocupa la población de M. peruvianus es de $119,9 \mathrm{~km}^{2}$ (Fig. 4). La población registrada está conformada por individuos en estado juvenil, adultos-maduros y adultos en estado de senescencia. En los individuos en estado de floración se observaron visitas continuas del colibrí cola corta Myrmia micrura (Trochilidae) lo cual evidencia una relación ecológica entre ambas especies no reportada anteriormente (Molina-Moreira 2017). Los frutos de esta especie suelen ser consumidos por el venado de cola blanca Odocoileus peruvianus (Cervidae), un mamífero muy común dentro de la Reserva Arenillas, del que se evidenciaron muchas huellas en el sector y se observaron individuos en áreas cercanas.

El ecosistema Espinar Litoral estudiado en la Reserva Arenillas, está dominado por la familia Cactaceae, entre las especies más abundantes se encuentran Pilosocereus lanuginosus y Armatocereus cartwrightianus con una

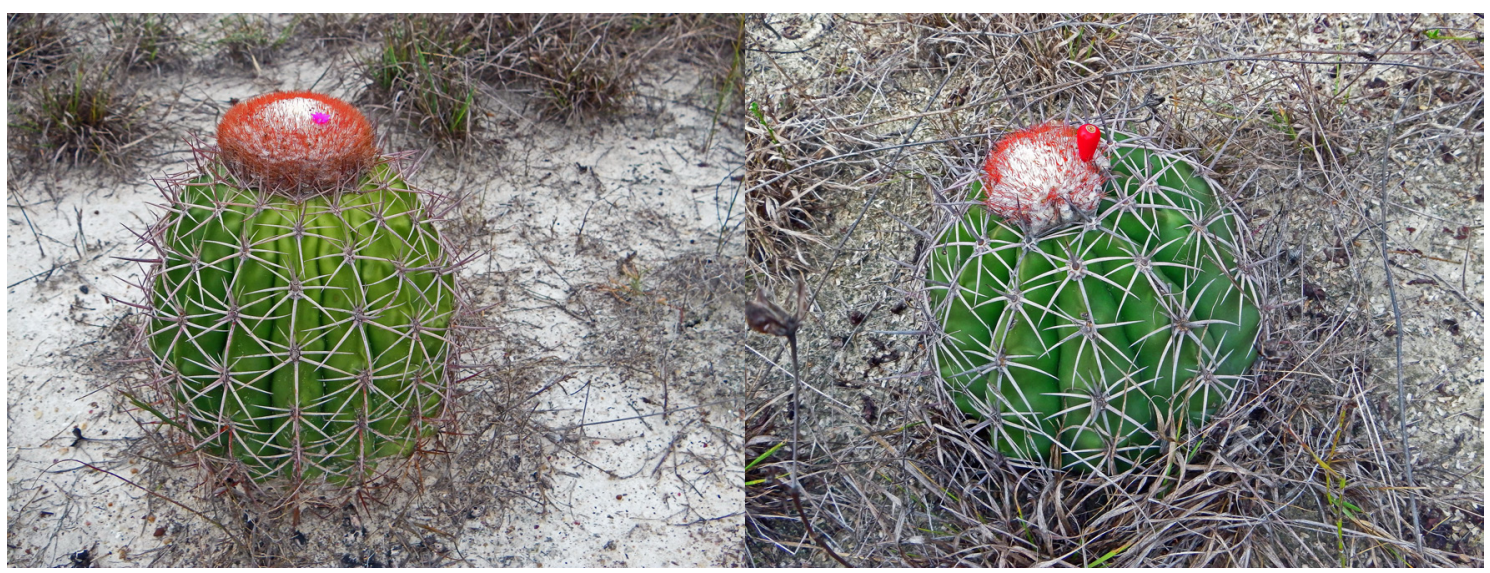

Figura 5 - Individuos de Melocactus peruvianus. Foto: Natalia Molina-Moreira

Figure 5 - Individuals of Melocactus peruvianus. Photo: Natalia Molina-Moreira 
densidad de 1.807 y 40 individuos por hectárea respectivamente. Otras especies de cactáceas registradas son: Hylocereus monacanthus, Praecereus euchlorus subsp. diffusus, Opuntia quitensis y Opuntia stricta. Entre las especies leñosas asociadas están Prosopis juliflora, Bursera graveolnes y Maytenus octogona, un arbusto siempreverde endémico de la región tumbesina y Galápagos (Molina-Moreira 2017).

\section{Modelamiento predictivo}

El modelamiento realizado para esta especie tuvo un nivel de aceptación bastante óptimo (Fig. 3). El valor de sensibilidad obtenido en el test data fue de 0.987 , lo cual indica que el $98,7 \%$ de las localidades observadas/datos de presencia estarían incluidos en la predicción. El valor de AUC obtenido en el modelamiento final fue de 0.979 (97,9\%), lo cual indica un nivel de confianza igualmente aceptable. De las variables utilizadas, nueve variables con valores superiores al $1 \%$ contribuyeron con el modelamiento, lo cual representa el 96,7 \% de la varianza explicada (Tab. 1). Las variables ambientales con mayor influencia para la distribución de $M$. peruvianus fueron: Precipitación del cuatrimestre más seco $(56,8 \%)$ y Precipitación anual (20\%). Otras variables de mayor y menor importancia se señalan en la Tabla 1.

\section{Estado de conservación}

Esta especie ha sido declarada como extinta en el Ecuador en varias publicaciones, debido a la falta de registros y porque se estima que su estado poblacional sería de 60 individuos/hectárea (Jørgensen \& León-Yánez 1999; Loaiza et al. 2009; Madsen \& Montúfar 2011). Se considera que la masiva creación y proliferación de camaroneras en la provincia de El Oro ha sido la principal causa para su extinción (Cerón et al. 2006). Otro factor que ha reducido y fragmentado el hábitat de esta especie es la redefinición de los límites de la Reserva Ecológica Arenillas establecida mediante el Decreto Ejecutivo 1208 (R.O. No. 342, 12 de Julio del 2012), ocasionando la pérdida de 3.912 hectáreas que en poco tiempo fueron deforestadas y convertidas en tierras agrícolas.

Si bien el nuevo registro de $M$. peruvianus reportado once años después del registro realizado por Cerón et al. (2006), nos permite confirmar que esta especie no está totalmente extinta en el Ecuador, se considera que la reducción y fragmentación de su hábitat, así como su reducido tamaño poblacional, ubicarían a esta especie dentro de la categoría de especies en Peligro Crítico de Extinción (CR), en base a los criterios A1ac y B1ab. Según los datos obtenidos en el presente registro, la población reportada se encuentra ubicada en un pequeño sector de Espinar Litoral (Figs. 3 e 4), el cual probablemente es uno de los últimos relictos que quedan de este ecosistema en la costa ecuatoriana.

\section{Discusión}

Las especies del género Melocactus tienen una distribución restringida en el Ecuador. $M$. bellavistensis se encuentra presente en la cuenca hidrográfica del valle de Catamayo, dentro de la provincia de Loja (Ecuador) (Loaiza 2008, 2010), y $M$. peruvianus habita solamente dentro de un pequeño relicto de bosque de Espinar Litoral, dentro de la Reserva Ecológica Arenillas en la provincia de El Oro (Molina-Moreira 2017).

La distribución de M. bellavistensis, tal y como ha sido reportada anteriormente, ocurre principalmente en los márgenes laterales y áreas de bosque cercanas a la cuenca hidrográfica del río Catamayo (Fig. 4), por lo cual su supervivencia se encuentra íntimamente relacionada a la conservación de dicho ecosistema. Lamentablemente, las formaciones vegetales en las cuales se encontraba ampliamente distribuida esta especie han ido desapareciendo gradualmente, además de que han perdido totalmente su flora original, siendo reemplazadas por extensas zonas agrícolas, con la consecuente introducción de especies exóticas. Como medidas de conservación, es necesario implementar programas de manejo que permitan la reintroducción de esta especie en su hábitat y su uso sustentable como planta ornamental, mediante la reproducción de plantas en viveros. Otra alternativa es implementar programas de educación ambiental para informar y concientizar a la población sobre la importancia ecológica que tiene esta especie, debido a su función como reservorio natural de agua para el bosque seco, contribuyendo con ello a la conservación de otras especies de flora y fauna nativas del valle de Catamayo.

En el caso de M. peruvianus, los principales problemas que amenazan las poblaciones de esta especie son los vehículos de carga y logística de las camaroneras que constantemente circunvalan sobre la vegetación del Espinar Litoral, además de la presencia y acumulación de depósitos con todo tipo de materiales y la quema de basura. Debido a que no existen hitos de la Reserva Arenillas que 
delimiten el área, una medida de conservación que se debe implementar para asegurar la sobrevivencia de esta población a largo plazo, es establecer zonas de amortiguamiento entre las camaroneras y el Espinar Litoral. Esta zona de amortiguamiento evitará que la población de $M$. peruvianus, esté directamente expuesta a las actividades humanas.

Otra alternativa de conservación viable para esta especie es el manejo ex situ, mediante la colaboración de un jardín botánico o viveros particulares (Hultine et al. 2016), se podría establecer y mantener un banco permanente de semillas, así como una colección de individuos vivos, que aseguren la conservación de la variabilidad genética de la población. Como parte de esta estrategia, se podría buscar obtener una ordenanza municipal que asegure la conservación de la población natural existente en la Reserva Arenillas como fuente segura y constante de semillas, lo cual será esencial para un programa de reintroducción de esta especie en su hábitat natural. Esta iniciativa podría incluir también la realización de una campaña nacional/internacional de donación de semillas y ejemplares de $M$. peruvianus dirigida a coleccionistas de cactus que pueden tener individuos de esta especie como parte de su colección.

La colaboración de entidades del estado ecuatoriano es algo prioritario para la conservación de ambas especies. Un paso fundamental sería la emisión de una ordenanza ambiental que prohíba totalmente la extracción ilegal de plantas de su hábitat natural, para ser comercializadas posteriormente. Por último, consideramos que la creación de un jardín botánico de cactáceas en el Ecuador, sería una buena estrategia para trabajar por la conservación de las especies más amenazadas y en grave riesgo de extinción.

\section{Literatura Citada}

Anderson EW (2001) The cactus family. Timber Press, Portland. 776p.

Britton NL \& Rose JN (1922) The Cactaceae: descriptions and illustrations of plants in the cactus family. Vol. 3. The Carnegie Institution of Washington. Washington DC. 258 p.

Cerón CE, Reyes CI \& Vela C (2006) Características botánicas de la Reserva Militar y Ecológica Arenillas, El Oro-Ecuador. Cinchonia 7: 115-130.

Fernández-Alonso JL \& Xhonneux G (2002) Novedades taxonómicas y sinopsis del género Melocactus Link \& Otto (Cactaceae) en Colombia. Revista Académica Colombiana de Ciencias 26: 353-365.
Hultine KR, Majure LC, Nixon VS, Arias S, Búrquez A, Goettsch B, Puente-Martínez R \& ZavalaHurtado JA (2016) The role of botanical gardens in the conservation of Cactaceae. Bioscience 66: 1057-1065.

Hunt D, Taylor N \& Charles G (2006) The new cactus lexicon. DH Books, Milborne Port. 526p.

Jørgensen PM \& León-Yánez S (1999) Catalogue of the vascular plants of Ecuador. Monographs of Systematic Botany of the Missouri Botanical Garden 75: i-viii,1-1182.

Kvist LP \& Moraes M (2006) Plantas psicoactivas. In: Moraes M, Øllgaard B, Kvist LP, Borchsenius F \& Balslev H (eds.) Botánica económica de los Andes Centrales. Universidad Mayor de San Andrés, La Paz. Pp. 294-312.

Lambert SM, Borba EL, Machado MC \& Da Silva SC (2006) Allozyme diversity and morphometrics of Melocactus paucispinus (Cactaceae) and evidence for hybridization with M. concinnus in the Chapada Diamantina, north-eastern Brazil. Annals of Botany 97: 389-403.

Loaiza CR (2008) Distribución y estado poblacional de Melocactus bellavistensis (Cactaceae), con notas sobre su proceso de floración y ecología reproductiva en el valle de Catamayo, provincia de Loja. Arnaldoa 15: 31-40.

Loaiza CR, Aguirre ZH \& Jadán O (2009) Estado del conocimiento actual de la familia Cactaceae en el Ecuador. Boletín de la Sociedad Latinoamericana y del Caribe de Cactáceas y otras Suculentas 6: 11-22.

Loaiza CR (2010) Análisis biogeográfico de Melocactus bellavistensis en la región sur del Ecuador: aspectos taxonómicos, distribución y conservación. Cinchonia 10: 106-118.

Machado MC (2009) The genus Melocactus in eastern Brazil: part I. an introduction to Melocactus. Cactus World 27: 5-20.

Madsen JE (1989) Cactaceae. In: Harling G \& Anderson L (eds.) Flora of Ecuador 35: 1-79.

Madsen JE (2002) Cactus en el sur del Ecuador. In: Aguirre Z, Madsen JE, Cotton E \& Balslev H (eds.) Botánica austroecuatoriana: estudios sobre los recursos vegetales en las provincias de El Oro, Loja y Zamora Chinchipe. Ediciones Abya-Yala, Quito. Pp. 289-303.

Madsen JE \& Montúfar R (2011) Cactaceae. In: LeónYánez S, Valencia R, Pitman N, Endara L, Ulloa C \& Navarrete H (eds.) Libro rojo de las plantas endémicas del Ecuador. 2a ed. Publicaciones del Herbario QCA, Quito. Pp. 249-252.

Molina-Moreira N (2017) Biodiversidad y zonación de los ecosistemas de la Reserva Ecológica Arenillas, Ecuador. Tesis de Doctorado. Universidad Nacional Mayor de San Marcos, Lima. 192p. 
Ostolaza C (2014) Todos los cactus del Perú. Ministerio del Ambiente, Lima. 538p.

Ostolaza C \& Loaiza C (2017a) Melocactus bellavistensis. The IUCN Red List of Threatened Species 2017: e.T151963A121515569. Available at <http://dx.doi.org/10.2305/IUCN.UK.2017-3. RLTS.T151963A121515569.en>. Access on 15 January 2018.

Ostolaza C \& Loaiza C (2017b) Melocactus peruvianus. The IUCN Red List of Threatened Species 2017: e.T152665A121544297. Available at <http:// dx.doi.org/10.2305/IUCN.UK.2017-3.RLTS. T152665A121544297.en>. Access on 15 January 2018.

Phillips SJ, Anderson RP \& Schapire RE (2006) Maximum entropy modeling of species geographic distributions. Ecological Modeling 190: 231-259.

Phillips SJ \& Dudík M (2008) Modeling of species distributions with Maxent: new extensions and a comprehensive evaluation. Ecography 31: 161-175.

Taylor NP (1991) The genus Melocactus (Cactaceae) in Central and South America. Bradleya 9: 1-80.

Taylor NP \& Zappi DC (2004) Cacti of eastern Brazil. Royal Botanic Garden, Kew. 511p.

Valencia RN, Cerón C, Palacios W \& Sierra R (1999) Las formaciones naturales de la Sierra del Ecuador. In: Sierra R (ed.) Propuesta preliminar de un sistema de clasificación vegetal para el Ecuador Continental. Proyecto INEFAN/GEF - BIRF y EcoCiencia, Quito. Pp. 79-108.

\section{Registros de localidades:}

\section{Melocactus bellavistensis}

1. Hacienda Algarrobera (Gonzanamá / Loja), 1203 msnm (679468 N, 9550324 E) AAU - 61154. 2. Cercanías del aeropuerto de Catamayo (Catamayo / Loja), 1250 msnm (679490 N, 9561382 E) AAU - 63971, 64153. 3. Hda. Algarrobera (Catamayo / Loja), 1399 msnm (670229 N, 9557714 E) AAU - 75913. 4. Vía Catamayo - Gonzanamá, 10 km al sureste de Catamayo (Gonzanamá / Loja), 1523 msnm (677621 N, 9552171 E) K - 89131. 5. Km. 5 - 11 de la carretera Catamayo - El Tambo (Catamayo / Loja), 1281 msnm (683174 N, 9552159 E) AAU - 7446. 6. Christian R. Loaiza S. / Base de datos personal (78 localidades / registros).

\section{Melocactus peruvianus}

1. $1 \mathrm{~km}$ al sur de Huaquillas, cerca del límite fronterizo con Perú (Huaquillas / El Oro), 10 msnm (585988 N, 9613135 E) MO - 2519. 2. Reserva Ecológica Arenillas (Huaquillas / El Oro), 2 msnm (593336 N, 9617489 E) QAP - 46670. 3. Ecosistema de Espinar Litoral, Camaronera Cayancas (Huaquillas / El Oro), 6 msnm (593478 N, 9617505 E) Registro visual / No colectado. 